\title{
Admissions During the COVID-19 Pandemic: Navigating an Altered Landscape for Successful Selection of Future Healthcare Providers
}

\author{
Andrea N. Belovich ${ }^{1} \cdot$ Ingrid Bahner $^{2} \cdot$ Giulia Bonaminio $^{3} \cdot$ Anthony Brenneman $^{4} \cdot$ William S. Brooks $^{5} \cdot$ Cassie Chinn $^{6}$. \\ Nehad El-Sawi ${ }^{7} \cdot$ Michele Haight $^{8} \cdot$ Sandra B. Haudek ${ }^{9} \cdot$ Robert J. McAuley $^{10} \cdot$ Douglas McKell $^{11,12} \cdot$ Rebecca Rowe $^{13}$. \\ Tracey A. H. Taylor $^{10} \cdot$ Mark D. Slivkoff $^{1} \cdot$ Richard C. Vari $^{14}$
}

Accepted: 5 February 2021 / Published online: 23 February 2021

(c) International Association of Medical Science Educators 2021

The Fall 2020 Web Seminar Series of the International Association of Medical Science Educators (IAMSE) was entitled "Admissions During the COVID-19 Pandemic: Navigating an Altered Landscape for Successful Selection of Future Health Care Providers.” This abbreviated series' overall goal was to provide faculty involved in health science program admissions with hands-on tips and insight into strategies developed to ensure the continuity of a rigorous and equitable admissions process in response to unparalleled changes brought on by the SARS-CoV-2 pandemic. From September 3, 2020, to September 17, 2020, this series was broadcast live by leading experts in three weekly webinars to worldwide audiences at academic institutions.

\section{Medical School Admissions in the Time of COVID-19: Maintaining the Integrity of Holistic Review}

Presenters: Christina J. Grabowski of the University of Alabama at Birmingham School of Medicine, Birmingham, AL, USA; Leila E. Harrison of the Washington State

Andrea N. Belovich

abelovich@idahocom.org

Idaho College of Osteopathic Medicine, Meridian, ID, USA

2 Morsani College of Medicine, University of South Florida, Tampa, FL, USA

3 University of Kansas School of Medicine, KS, Kansas City, USA

4 University of Iowa Carver College of Medicine, Iowa City, IA, USA

5 University of Alabama at Birmingham School of Medicine, Birmingham, AL, USA

6 International Association of Medical Science Educators, Huntington, WV, USA
University Elson L. Floyd College of Medicine, Spokane, WA, USA.

The presentation by Drs. Grabowski and Harrison started with detailing the impact of COVID-19 on medical school applicants whose premedical experiences were altered when the pandemic began in March 2020. Faculty members transitioned lectures and labs from in-person to remote environments. Access to clinical experiences was restricted, and there were delays and cancellations of the Medical College Admissions Test (MCAT). In addition to its effects on academics, the pandemic also impacted applicants' personal lives. Many suffered job and health insurance losses, impaired health and wellness, and forced relocation due to university closures. The presenters cautioned medical schools to consider each of these impacts when preparing for upcoming interviews.

As coursework had to be transitioned to online learning across nearly all health sciences schools, interviews also were transitioned to virtual platforms to accommodate students who were unable to travel, as well as to prioritize the health and safety of all parties even in the absence of a general lockdown. Admissions staff utilized new tools

Des Moines University Medicine \& Health Sciences, Des Moines, IA, USA

8 Sam Houston State University College of Medicine, Huntsville, TX, USA

9 Baylor College of Medicine, Houston, TX, USA

10 Oakland University Beaumont School of Medicine, Auburn Hills, MI, USA

11 Elms College, Chicopee, MA, USA

12 Bay Path University, Longmeadow, MA, USA

13 University of New England College of Osteopathic Medicine, Biddeford, ME, USA

14 Virginia Tech Carilion School of Medicine, Roanoke, VA, USA 
and technologies to restructure interview day events and produced videos and virtual tours to showcase campuses. These changes required additional training of interviewers and everyone involved with the admission process.

Dr. Grabowski explained that while the pandemic has impacted every student, students from disadvantaged and marginalized populations have faced additional challenges. Several of the concerns raised included equitable access to technology and fast internet connections as well as a lack of availability of private space at home for study and interviews. She also addressed concerns that the virtual environment may have led to a loss of accommodations for students with disabilities. For example, masks make it impossible for the hearing impaired to read lips.

Mitigating unintended consequences on applicants and school employees has involved changes to the application and screening processes and committee and interviewer training. As Dr. Harrison explained, many schools' secondary applications now include a separate essay which permits applicants an opportunity to share how the pandemic has impacted them. This change assists reviewers in gaining further insight into the experiences of applicants during the pandemic. She also discussed the interviewers' training, focusing on the importance of avoiding biases against applicants based on where they are interviewing (e.g., in their cars) or what they may be wearing during the interviews. Her staff has improved communication with applicants and pre-health advisors to alleviate anxieties and remain as transparent as possible during the admissions cycle.

Dr. Grabowski concluded the discussion by stressing the importance of holistic review of applicants, with increased individualized consideration of experiences, attributes, and metrics in the context of the applicant's lived experiences. She emphasized the benefits of focusing on competencies rather than the time and duration of experiences. Finally, she identified several opportunities highlighted by the pandemic: (1) applicants may showcase attributes such as resilience and adaptability, (2) expenses and burden for travel may be reduced, (3) schools may save on cost for travel and events, (4) awareness of bias and impact on disadvantaged or marginalized populations is heightened, (5) new technologies are available for use, (6) stakeholders from remote campuses/locations can be engaged in the interview process, and (7) creative thinking about how we do what we do is promoted.

\section{PA and PT Admissions in the Time of COVID-19: A Panel Discussion}

Presenters: Erika Brooks of the Physician Assistant Education Association, Washington D.C., USA; Thomas O'Shea of the University of Iowa Carver College of Medicine, Iowa City,
IA, USA; Jeremy Turkall of the University of South Florida Morsani College of Medicine, Tampa, FL, USA.

Ms. Brooks began the webinar with a discussion of how the national Physician Assistant Education Association (PAEA) supports its members during the pandemic. She explained that the PAEA provides members with resources to adapt to the admissions process, maintain its integrity, and create awareness about implicit bias in the admissions process. The PAEA has helped schools transition to new virtual environments by hosting virtual fairs for campus visits, managing the integrity of interviews and the application process, and providing resources for matriculating students to help create a sense of belonging. As the PAEA organization also plays a fundamental role in supporting the Centralized Application Service to Physician Assistants (CASPA), Ms. Brooks explained how the application process to physician assistant programs has also evolved in response to the pandemic. There is now a COVID-19 impact essay requirement and an allowance for unofficial transcripts from applicants who may be experiencing difficulty obtaining official transcripts from academic institutions.

The PAEA has also been actively surveying its member schools since the pandemic began. The survey results revealed that $74 \%$ of member schools have eliminated in-person interviews, $64 \%$ are offering virtual tours, and $27 \%$ are paying particular attention to the impact essay. Approximately $13 \%$ of programs have not made any changes to their admissions process.

Dr. O'Shea continued the webinar by addressing how his home institution has altered its admissions process in response to the pandemic. In order to fulfill the mission of the program it was necessary to re-evaluate the selection process in the face of COVID-19-related problems. The admissions committee pondered questions related to academic issues such as grade point average (GPA) requirements, acceptance of pass/fail courses and online coursework, graduate record examinations (GRE) requirements, and recommendation letters. Concerns of the committee included applicants' inability to obtain the required $1000 \mathrm{~h}$ of volunteer work, applicants' impaired access to school computers to submit online applications through CASPA, as well as how to give applicants the best possible virtual interview experiences. According to Dr. O'Shea, his institution decided that while the general interview process itself did not require change, it was crucial for the program to alter some of the variables considered for admission. The program began to accept pass/fail graded courses towards requirements and permitted GRE scores to be self-reported. The number of volunteer healthcare hours was also reduced from 1000 to 500 , and admission interviews were conducted virtually. 
The panel discussion was concluded by Jeremy Turkall, who discussed updates and changes to the Doctor of Physical Therapy (DPT) program at his institution. He described modifications related to PTCAS, the Physical Therapy Centralized Application Service. These changes included launching the application service 3 weeks earlier than normal to allow applicants more time to prepare as well as increasing the number of maximum recommendation letters from four to five in order to capture additional insight from individuals who know the applicants. Like CASPA, a COVID-19 impact question was also added to the application, although was optional for the Physical Therapy Centralized Application Service (PTCAS). Mr. Turkall also explained that in response to the pandemic, pass/fail courses and online courses were now accepted towards the prerequisites and the requirements for observation hours and two letters of recommendation from licensed physical therapists were waived. However, GRE scores remained a requirement, with a due date of November 2020. In the case of the University of Florida DPT Program, interviews were not affected since the program does not carry out interviews as part of the application process.

\section{Navigating the Effects of COVID-19 on the 2020-2021 Residency Application Cycle}

Presenters: Maya M. Hammoud, Versha Pleasant, and Keli Santos-Parker of the University of Michigan, Ann Arbor, MI, USA.

In this final presentation of the 2020 Fall mini-series, the focus turned towards graduate medical admissions for residency training. Drs. Hammoud, Pleasant, and SantosParker, all from the University of Michigan, discussed the implications of COVID-19 on the medical residency application cycle and outlined some of the actions applicants and programs might take to mitigate its harmful effects.

Dr. Hammoud began the webinar by discussing how COVID-19 has affected and will potentially continue to affect the applications, screening, interviews, and postinterview processes for residency programs. Residency programs will still use the Electronic Residency Application Service (ERAS), but the timelines for submission have been altered. Program directors will accept applications earlier (October 21, 2020) than in previous years. Additionally, as the number of applications to residency programs continues to rise, further challenges are posed as applications continue to outnumber the number of residency positions. Programs will review the full application, though the United States Medical Licensing Exam (USMLE) Step 2 scores and clinical skills exams for international students may be absent or delayed. According to Dr. Hammoud, program directors will still need to decide how to handle incomplete applications. It was also pointed out that the reduced timeline and increased number of applicants could prove problematic, as program directors are concerned about being unable to complete holistic reviews for each applicant. Residency programs will conduct virtual interviews, which presents more challenges to the applicants and program directors based on new technological demands and the lack of face-toface interaction. Remote interviews increase the difficulty of ranking candidates, sparking concern that program directors may be more apt to rank students graduating from their home institutions higher than students graduating from other medical schools.

Dr. Hammoud emphasized that the current pandemic amplifies problems already inherent in the residency application process. She and others have recently published a paper in the Journal of the American Medical Association (JAMA) that addresses the following proposed changes to the process [1]:

- Adjust the residency application timeline

- Modify application requirements

- Encourage holistic review

- Limit the number of applications

- Expand program information available to applicants

- Improve the quality of information programs receive

- Temporarily make exceptions to the National Resident Matching Program (NRMP) all-in policy

- Cap the number of interviews a student can accept

- Implement preference-signaling mechanisms

Dr. Hammoud concluded by calling for all interested stakeholders to consult the recent guidelines for their specialties, available via the Association of American Medical Colleges (AAMC) [2].

Dr. Versha Pleasant continued the webinar with a discussion of virtual experiences and the impact on underrepresented medical students during COVID-19. She first discussed the guidelines of the American Medical Association (AMA) regarding underrepresented students and residents during COVID-19, highlighting the following points in their report [3]:

- COVID-19 impacting all areas of society but not equally

- Additional stressors of structural racism

- Disruptions from COVID-19 amplify underlying inequalities

- Responsibility of the AMA to advocate for all medical students and reverse the historic active exclusion of racially marginalized groups, specifically Blacks, Latinx, and Native Americans 
Dr. Pleasant reported that some of this education inequity involves the shift to virtual platforms, which requires access to technology and dedicated spaces. Loss of enrichment opportunities, including shadowing, research, and global experiences, is also concerning. Furthermore, program directors must consider geographical inconsistencies and limitations in obtaining letters of recommendation and the ability to participate in away rotations. She emphasized the potential emotional toll or "weathering effect" these challenges may have on underrepresented minorities. To address these issues, the University of Michigan hosted a workshop in August of 2020. The organizers dedicated this 90-min workshop to sharing the university's culture with an emphasis on diversity and inclusion. Underrepresented minority (URM) department members participated and shared their residency interview experiences. The school plans to provide a follow-up leadership conference. Dr. Pleasant concluded her portion of the webinar by reiterating that the new climate has required us to be flexible and creative. Despite these changes, we must remain committed to diversity and equity.

Dr. Santos-Parker, who holds a PhD in mathematics and is currently a University of Michigan medical student in the process of applying to residencies, concluded the webinar with a focus on student perspectives. He explained that the pandemic has coincided with the normal process and timing of selecting residencies, identifying mentors, and attending conferences. He elaborated on the usual stressors of the application process, placing emphasis on challenges that the pandemic has introduced to securing away rotations, which are often used by applicants to demonstrate their preference for a particular residency program. He spoke of innovations, such as virtual events, that may help fill this component of the application process. Other preferencesignaling mechanisms include token systems and limiting the number of applications and interviews, all of which are currently being utilized by applicants.

Dr. Santos-Parker then addressed the advantages and disadvantages of virtual interviews. While they are more cost- and time-efficient, virtual interviews, of course, lack in-person social interactions, making it more difficult for applicants to assess the local environment of the program.
Applicants also have concerns about the possibility of increased application and interview inflation due to the convenience of virtual interviews. Dr. Santos-Parker noted that, ideally, applicants should limit their applications to fewer programs of genuine interest. However, he acknowledged the dilemma many students face in wishing to apply broadly to increase the odds of acceptance. Dr. SantosParker voiced concern that if widely practiced, applying to a multitude of programs will diminish the holistic review of applications due to increased volume of applicants. He urged applicants to apply to programs intentionally, communicate with mentors, and promptly decline interview offers if not interested. Finally, he concluded the webinar by mentioning potential avenues for change that may mitigate these stressors and pressures, such as coordination between residency programs regarding interviews and the use of predetermined dates for notifying applicants that programs have offered them interviews.

We wish to thank all presenters and the audience who made this series such a success!

\section{Declarations}

Conflict of Interest The authors declare that they have no conflicts of interest.

\section{References}

1. Hammoud MM, Standiford T, Carmody B. Potential implications of COVID-19 for the 2020-2021 residency application cycle. JAMA. 2020;324(1):769-86. https://doi.org/10.1001/ jama.2020.8911.

2. Specialty Response to COVID-19. Association of American Medical Colleges. 2020. https://students-residents.aamc.org/ applying-residency/article/specialty-response-covid-19/.

3. Protecting underrepresented students and residents during COVID-19. American Medical Association. 2020. https:// www.ama-assn.org/delivering-care/public-health/protectingunderrepresented-students-and-residents-during-covid-19.

Publisher's Note Springer Nature remains neutral with regard to jurisdictional claims in published maps and institutional affiliations. 